\title{
The Dissection of Three-Dimensional Geographic Information Systems
}

\author{
Yong Xue ${ }^{1,2}$, Min $\mathrm{Sun}^{3}$,Yong Zhang ${ }^{4}$ and RenLiang Zhao ${ }^{5}$ \\ ${ }^{1}$ LARSIS, Institute of Remote Sensing Applications, Chinese Academy of Sciences, \\ Beijing, 100101, PR China \\ ${ }^{2}$ School of Informatics and Multimedia Technology, \\ University of North London, 166-220 Holloway Road, London N7 8DB, UK \\ $\{y \cdot x u e @ u n l \cdot a c \cdot u k\}$ \\ ${ }^{1}$ Institute of RS \& GIS, Peking University, Beijing, 100871, PR China \\ ${ }^{4}$ Institute of Computer technology, Tsinghua University, Beijing, 100871, PR China \\ ${ }^{5}$ Institute of Surveying and Mapping, Central South University, Changsha, 410083, \\ PR China
}

\begin{abstract}
In this paper we dissected the 3-dimensional Geographic Information Systems (3D GISs) and disambiguated some points such as (1) 3D GIS should be a component of GIS, it's a subsystem; (2) data modelling is not the main obstacle to its development; (3) it's no necessary and also very difficult for 3D GIS to replace GIS; (4) the main developing direction of 3D GIS should be special subsystems, that is, 3D GIS research must be based on relative application areas.
\end{abstract}

\section{Introduction}

We are living in a true 3-dimensional (3D) world. The environment and almost all artificial objects have 3-demensional sizes. However, as so far, it's difficult to establish a uniform data model to represent the environment and the objects. In GIS, objects are represented using its projections on 2D plane. Although this kind of representation could solve many problems, it is still very difficult to represent and process the geometry and attributes for third spatial dimension. For example, GIS can't represent real 3-dimensional nature and artificial phenomena in geosciences area, such as in geology, mining, petroleum, etc. Many research works about 3D GIS have been carried out in order to remedy such defects in solving true 3-dimensional problems. It has been found that it is almost impossible to establish a 3D GIS that has similar functions as those in current GISs, especially for the processing of complex 3dimensional spatial relationships.

Data modelling of 3D objects is one of the most difficult problems. As data model is the fundament to the establishment of GIS, research works on 3D GIS have been mainly focused on data model. So far, there isn't a perfect data model found. The great difficulties for 3D GIS research could be summarized in three points: 
1. Try to find a completely new 3-dimensional data model to substitute GIS vector data model. Third dimensional value $Z$ not only changes from attribute data to spatial data, i.e.: $(\mathrm{X}, \mathrm{Y}): \mathrm{Z} \rightarrow(\mathrm{X}, \mathrm{Y}, \mathrm{Z})$ but also a new volume element is added in addition to three elements such as point, line and face;

2. Try to represent various 3-dimensional objects in one data model;

3. Try to represent objects, especially complex real objects, in high precision.

Most research works on data modelling have been to establish a common 3dimensional data model based on 3D GIS as a common platform for real world complex objects, including geology, mining, petroleum, cataclysm, ocean, atmosphere, and urban environments. However, no significant progresses have been made so far. In this paper, the concept and applications of 3D GIS will be addressed first. Data modelling will be studied and the further development of 3D GIS will be discussed at the end.

\section{3D GIS CONCEPT AND APPLICATIONS}

\subsection{Applications of 3D GIS}

We could find GIS applications almost in any areas that relate to spatial data. Object scales might vary from 1:500 to 1:1,000,000, even larger or smaller. That's in such scale areas, we could find actual objects represented by GIS data model, e.g.: in 1:500 scale a polygon might represent a house, while in 1:1,000,000 scale and a polygon might represent a city area. But in 3D GIS, the case changes a lot. We could analyse this question from GIS data models.

GIS is mainly established on vector data model. In this model, line is composed of points and face is composed of lines.

- Point element $P(x, y, z)$ may represent any single object occupying one spatial point location, and also an object occupying 2-dimensional spatial areas, e.g.: streetlight, road crossing, station or even a whole city;

- Line element $L\left(P_{1}, P_{2}, P_{3}, \ldots\right)$ may represent any object that occupies 1dimensional or 2-dimensional space, e.g.: pipelines, roads and rivers;

- Face element $S\left(L_{1}, L_{2}, L_{3}, \ldots\right)$ may represent any object that occupies 2dimensional space, from single position to small area or even a very large space, e.g.: house, square, city and whole country terrain.

Therefore, GIS vector data model could represent random size objects in plane, and could be used in many spatial related applications. In 3D GIS, space is extended from $2 \mathrm{D}$ to $3 \mathrm{D}$. Volume element has to be introduced in both vector model and raster model [14], [15], [16], [20].

If we consider volume element represented by components of point, line and face elements, i.e.: $V(R(P, L, S))$, then in theory, volume $V$ could be used to represent any 
objects in Euclid space with random dimension $(\mathrm{d}<=3$ ) and random size. However, such representation is limited in practice. For example,

- City environment: in city environment, the main objects are artificial objects (including constructers, bridges, etc.). Their third dimensional sizes are usually in a certain range, e.g.: from 1 meter to 100 meters. If we use scale 1:10,000 to represent one constructer with $100 \mathrm{~m}$ in its width, length and height, its map size is only $1 \mathrm{~cm} \times 1 \mathrm{~cm} \times 1 \mathrm{~cm}$. Such a size representation is much similar to its plane representation, and for many smaller objects, it already loses 3D representation significance. In addition, third information significance of 3D city object would change with scale. For example, it has significance to represent one balcony in 1: 1,000. But in 1: 10, 000 scale, representation of whole building in detail would lose significance. Therefore, to city environment, 3D GIS spatial data modelling should be limited to large scale, e.g. no large than 1:10,000.

- Ocean and atmosphere environments: these two environments are reverse to city environment. Information is captured usually in small scales, and artificial objects would lose significance. Information represented usually only in small scales would have significance (e.g.: smaller than 1: 100,000), e.g. temperature and humidity distributing; clouds and ocean flow excursion etc. At the same time, in such environments, medium distributing has continuity. It is difficult to model according individual object as in city environment. Therefore, in such environments, 3D GIS data modelling is not only limited to small scales, but also much different from city environment. Data modelling can't be established on individual object.

- Mining, geology, cataclysm and petroleum environments: in these environments, there are both nature objects and artificial objects. But if we review these environments, we could conclude two main cases. One related to resource exploring, which information capture is usually in large scales while with high precision. Another relates to macro geosciences research, such as: terra construction, cataclysm monitoring, etc., which information capture is mainly limited to small scales, similar to atmosphere and ocean environments. In present research works on 3D GIS, most works are related to resource exploring. Therefore, we could get a rough conclusion that in such environments, 3D GIS data modelling are mainly limited to large scales, but have great difference to city, atmosphere and ocean environments.

Now we can summarize a conclusion that 3D GIS data modelling not only has great differences in different environments, but also has great different spatial scale requirements in different environments. It is very difficult to find a kind of volume element in data modelling to meet many different object representations in various environments and various scales.

\subsection{D GIS Concept and its research contents}

The current GISs should be called 2D GISs because they are developed from plane map. They could only disposal actual 2D spatial information and their data models are 
mainly based on 2D vector elements, such as point, line and face. Deunis et al. defined GIS as a software system that contains function to perform input, storage, editing, manipulation, analysis and display of geographically located data [4]. Here geographically located data are referred to 2D spatial data because in GIS data model, third dimensional data is disposed as attribute data.

So far, there is no formal definition for 3D GIS. Different people have different considerations. Fritsch pointed out that main research work of 3D GIS is the integration of height information into 2D GIS [6]. Kofler considered that one major difference between 3D GIS and 2D GIS is the amount of data to be processed, and the second fundamental difference is the user interface [11]. Pigot pointed out that similar to $2 \mathrm{D}$ variants, 3D GIS should be capable to perform metric (distance, length, area, volume, etc), logic (intersection, union, difference), generalization, buffering, network (shortest way) and merging operations [19]. Pfound pointed out that today, most applications and data structures for 3D GIS are optimised for visualization [18]. They usually omit topological information in order to get a better performance. Actually, these hints could be found in many papers. These hints imply that 3D GIS is a new system that should be established on the base of 2D GIS. From there, we could define that 3D GIS is a software system that contains function to perform input, storage, editing, manipulation, analysis and visualization of three-dimensional geographical information data in one certain application area.

\subsection{Research content}

The majority of research works on 3D GIS are 3D data modelling and 3D data structure [6], [15], [16], [19], [20]. A few is on 3D visualization [11] and others are on 3D web GIS [13][26]. From the scientific view, 3D GIS research content must be larger. On the other hand, if we consider the above definition, 3D GIS research content may focus on following points:

- 3D data modelling base on actual applications;

- 3D data structure base on actual applications;

- 3D visualization technologies for actual applications;

- Huge multimedia data management;

- 3D technologies on web for actual applications.

These contents have strong relations with application areas. The majority of 3D GIS research works are carried on with the other research works for actual application. Hence such considerations would meet the practice needs. While the mainstream of 3D GIS research works seldom consider situations in actual application, this is the main reason why we can't see one 3D GIS used in practice beside some special software used in certain areas like petroleum or city. 


\section{Data Modelling in 3D GIS}

The final aim of data modelling is to truthfully represent the real world. However, because of limitation of theories and technologies, it is difficult to redivivus real world objects through data modelling in GIS. The data models could be classified into three levels or three types according to their representation precision:

Symbol model: in 2D GIS vector data model, point and line element could be considered as symbols on plane as a face is composed of lines. We can consider face is represented by line symbols because 2D GIS comes from map, and objects representation manners are not changed from map to GIS. 2D GIS vector data model can be considered as a symbol model and this model can represent objects location and its rough shape. Symbols are suitable to represent symbolic logic. This is the reason that $2 \mathrm{D}$ GIS vector data model could be easily used to represent spatial topology.

Basic model: a few spatial data, and predefined elements, such as points, lines, faces and bodies can be used to represent various objects, and could basically reflect object's shape, spatial extension and higher precision 3-dimensional spatial location. 3D GIS data models are mainly used for the representation of basic information of objects in real world. For example, tetrahedron models were used to represent irregular mine body [20] and CSG primitives were used to represent regular buildings [14]. In city environment, third dimensional spatial information can be added to construct 3D urban GIS models easily [7][9]. Today, the majority of data models available are belong to basic model category.

Refined model: a large number of spatial data are used in this kind of models. It is established mainly through interactive methods, and it is difference from above two kinds significantly. Many types of elements are used in this kind of data modelling, and these elements could be adjusted to meet modelling needs during modelling process. This kind of data model could reflect objects with high precision in spatial location, subtle spatial shape and vision characteristics, etc. It is mainly used in Virtual Reality GIS (VRGIS), especially in 3-dimensional landscape reconstruction based on GIS spatial data. As VR technology need very high precision representation of 3-dimensional objects (e.g.: construction, road bridges, trees, etc.), high precision are also requested in data capture and data modelling functions. Therefore, at the present time, this kind of data models could only be established using software packages like 3Dmax and AutoCAD. It is very difficult for GIS to perform this kind of data modelling.

Obviously, refined model needs very high precision representation. This may cause huge amount of data and modelling complexity. As different objects may have different characteristics, it is difficult to realize automatic data modelling in large quantities. Special modelling software packages must be used. Therefore, refined model is not suitable in 3D GIS and it is more suitable for VRGIS.

On the other hand, as symbol model can't be used in 3D GIS, basic models are usually used in 3D GIS data modelling. Basic model can still be classified into two types according its modelling purposes: 
1. Currency model: the aim of this kind of models is to represent various objects in different application areas in real world. The majority of present data models are belong to this category. For example, Molenaar put forward a 3D FDS data structure [15]. Pilouk et al. put forward a tetrahedron based data model [20]. Pigot put forward a topological data model [19]. Li put forward a CSG based data model [14], and etc. Although these data models have different advantages, there are still some defects in different degrees as currency models to represent various complex 3-dimensional objects in real world. The realisation of truly 3-dimensional GIS solutions is not yet in view [3].

2. Special model: it is established for certain application area. Early in 1989, Peter pointed out that elements in geosciences have a lot of communities [17]. It could be possible to establish one uniform geosciences data model. Raper discussed 3D data modelling for geosciences [21]. He pointed out that 3D data models have strong links with underground data samples and parameters. Turer pointed out that an exact model of the subsurface is not possible, simplified versions may be derived from geological and engineering data developed from exploration procedures, including borings, well tests, seismic exploration, and stratigraphic or sedimentological descriptions of depositional systems [25]. In city environment, Zlatanova used faces and nodes as main elements combining R-tree to establish a 3-dimensional topological data model on Web [26]. Gruen et al. developed a TOBAGO system using point sets to establish 3D data model by interactive method [8]. Tempfle put forward a 3D topological urban data model [24]. Koehl put forward a data modelling method based on CSG level structure [10] and Sun et al. put forward a 3D spatial data model based on surface partition [23].

Because special models disposal objects limited in certain application areas, data modelling is relatively simple. It is also easy to implement in practice. For example, using 3D surface modelling method, ArcView 3D analyst module could realize modelling to construction [5].

\section{Is there a perfect spatial data model for 3D GIS?}

\subsection{Perfect 3D GIS data model}

Pfund pointed out in his research work that a perfect 3D GIS data model should be vector data structure, boundary representation and topology [18]. Peter pointed out that boundary representation (B-rep) is a suitable modelling method for geosciences elements representation and operation when he did research works on one Geoscientfic Resource Management System [17]. Pigot had summarized 3D GIS modelling research works [19]. He pointed out that 3D GIS data modelling began with Molenaar's 3D FDS data structure and many others research works are developed on the base of it. Pigot himself put forward a 3D spatial data model based on manifold topology. This model is similar to 3D data models developed by Pilouk et al. [20]. 
Actually, Molenaar's 3D FDS is an extension of 2D GIS vector data model and it is a B-rep data model. B-rep data model is a kind of perfect data model in some degree. The reasons are:

- The basic elements are point, line, face and body. Point and line are the most basic elements, so it is easy to combine a 3D GIS with a 2D GIS;

- Amount of data is not large, and it is easy to establish data model and to manage;

- Easy to establish topology;

- Easy to realize visualization, and easy to do data operations;

But B-rep method also has some defects. It is difficult to verify representation validity, to perform Boolean operations, and to require complex algorithms for model generation [17]. However, if we don't pay too attention to verification of representation validity, it doesn't effect today's 2D GIS so much. Boolean operations are not so urgent and complex algorithms become simple to today's computer hardware. So we think B-rep data model is a kind of perfect 3D GIS data model.

\subsection{Questions about spatial topology relationships}

This question may raise many arguments, especially in 3D GIS. Topology representation is considered as the typical feature of GIS, but why do we have to represent topology relationships? Spatial topology could be established dynamically. The essence of this thinking is to calculate topology in real-time (e.g.: Voronoi diagram is a good structure). Actually, current 2D GISs could only represent a few topology relationships, and topology problems on 2D plane are still not solved very well [1].

Topology relationships representation usually limits the establishment of data model, and it also increase the complexity of data updating and operation. The topology relationships in 3D GIS could be calculated in real-time. AutoCAD might give us a good example. There are no any topology concepts in AutoCAD, but it could be used very well in industry, such as machine assemble. Bernhardsen pointed out that the essential difference between GIS and CAD becomes more and more blur [1]. By adding some attribute data, many CAD system vendors could effectively compete GIS market. Kofler used a CAD model and R-tree combined method in Vienna walking system [11]. Stoter also introduced a CAD model into GIS in order to solve the problem of 3D cadastral registration [22]. Koehl put forward CSG level model. His main purpose was to solve CAD models representation [10]. In VRGIS, data modelling mainly comes from 3Dmax or CAD.

\subsection{D GIS data modelling for geoscience}

Data models based on tetrahedron (or 3-simplex) could preferably be used to represent various elements in geosciences. In geosciences, most objects are natural objects not artificial ones. Lattuada and Raper put forward that 3-dimensional tetrahedron network is suitable for modelling in these areas [12]. Recently, we also 
did some research works in terra area, we reconstruct 3D geological body on the base of simulate artesian well data using tetrahedron network, and also reconstruct complex geological bodies on the constraint tetrahedron network. Tetrahedron model is not suitable to represent regular bodies, especially some bodies with arc shape, such as cylinder, cone and etc. Extra partition calculation and redundancy data will be introduced if tetrahedron is used to represent regular objects.

In city environment, almost all objects are artificial objects. Besides, it is not necessary to represent inner attributes of city objects in GIS. As majority of city objects are regular ones, they could be represented by some primitives like rectangle, triangle, cuboids, cylinder, cone, etc. or their combinations [10][15][23]. Some CSG primitives could not be represented by R-rep, but they could be deal with parameter expressions.

\section{3D GIS Development Directions}

Although the general 3D GIS system have not be progressed significantly because of the baffle in data modelling, the great progress have been got in Three-Dimensional City Model and Virtual Geographic Information System. These systems could provide very excellent visualization effects and interactive functions.

3D GIS should be designed as a subsystem of 2D GIS. Of course, it could run alone. The establishment of 3D GIS should be compatible with 2D GIS functions. It should have interface same as that for 2D GIS. And reducing 2D GIS functions would decrease much complexity in 3D GIS. On one hand, 3D GIS should have unique visualization interface as well as operation and analysis functions. Many systems are established on the base of GIS [9]. While in geology, mining, etc., although subsurface data is the principal, ground data is also important. For example, 2D data in GIS is usually the reference to underground works in resource mining process.

Therefore, It is a crucial point to set up a connect between 3D GIS and 2D GIS, we have several views as (1) Establish special SDE for 3D spatial data management; (2) Extend present 2D GIS data manage functions and make them compatible with 3D spatial information management; (3) Develop 3D GIS modules in component methods; (4) Establish data change interface between 2D to 3D.

Cambray et al. put forward a multidimensional geographical data model, through add some faces with different heights to solve this problem [2]. Their data model could only solve regular and protruding enveloped objects. Arc view 3D analyst is a well known 3D GIS module developed based on component. Haala et al. established a 3-dimensional city model by adding some extra information and changing 2D data to $3 \mathrm{D}$ data [9].

3D GIS has no great significance in many application areas, as in small scales space (e.g.: scales less then 1:10,000). There are great different spatial characteristics and requirements in city, geology, mining, ocean, atmosphere and other environments. It is impossible to establish a currency data model to these areas in theory. In fact, from the review of GIS software packages (e.g. Arc/Info, MapInfo, GeoMedia, AotoDesk), we would found that in these GIS software packages, 3D 
functions are limited to a few modules. For city environment, functions are limited to visualization and simple queries.

We conclude that 3D GIS development should be based on special application. The research should focus on application areas information representation and analysis. 3D spatial data is only a carrier. Data modelling should focus on representation integrality to special information. It should not simply emphasize precision and topology consistency of spatial data representation. At the time being, it is not necessary, and also very difficult to establish a currency 3D GIS platform.

\section{ACKNOWLEDGMENTS}

This publication is an output from the research project "Digital Earth" funded by Chinese Academy of Sciences. Also, Dr. Yong Xue should like to express his gratitude to Chinese Academy of Sciences for the financial support under the "CAS Hundred Talents Program" and "Initial Phase of the Knowledge Innovation Program".

\section{Reference}

1. Bernhardsen T. “Geographic Information Systems An Introduction”. John Wiley \&Sons Inc., New York, 1999.

2. Cambray B., Yeh T. S. "A Multidimensional (2D, 2.5D, 3D) GeoGraphical Data Model". Proceedings of the International Conference on Management of Data (COMAD'94), Bangalore, India, 1994, pp317-336.

3. Carosio A. "Three-Dimensional Synthetic Landscapes: Data Acquisition, Modelling and Visualisation". Photogrammetry week'99, 1999, pp293-302.

4. Deunis R. S., Arithur R. P., "Three-dimensional GIS for the earth sciences". In: Three dimensional applications in Geographical Information Systems, Taylor \& Francis, Edit by Jonathan F. Raper, pp 149-154, 1989.

5. Esri Company "ArcView 3D Analyst". http://www.esri.com

6. Fritsch D., "Three-Dimensional Geographic Information Systems -- Status and Prospects". Proceeding of International Archives of Photogrammetry and Remote Sensing, Vol. 31/4, ISPRS, Vienna, Austria, 1996, pp215-221.

7. Gruber M. "The CyberCity Concept from 2D GIS to the Hypermedia Database". Proceedings of UM3'98, International Workshop on Urban Multi-Media/3D Mapping, 1998, pp47-54.

8. Gruen A. "Tobago-A semi-automated approach for the generation of 3D building models". ISPRS Journal of Photogrammetry \& Remote Sensing, 53, 108-118, 1998.

9. Haala N., Brenner C. and Anders K. H. "Generation of 3D City Models From Digital Surface Models and 2D GIS". ISPRS, Vol.32, Part 3-4W2, 3D Reconstruction and Modelling of Topographic Objects, Stuttgart, 1997, pp68-75. 
10.Koehl M. "The Modelling Of Urban Landscapes." Proc of International Archives of Photogrammetry and Remote Sensing. Vol. XXXI, Part B4. Vienna, 1996, pp460-464.

11.Kofler M. "R-trees for Visualizing and Organizing Large 3D GIS Databases". PhD. thesis, Technischen Universität Graz, Graz, 1998.

12.Lattuada R., Raper J. "Applications of 3D Delaunay triangulation algorithms in geoscientific modelling". http://geog.bbk.ac.uk

13.Lee H.G., Kim K.H., Lee K., "Development of 3-Dimensional GIS Running on Internet”. IEEE, pp1046-1049, 1998.

14.Li R.X., "Data Structure and Application Issues in 3-D Geographical Information Systems". Geomatics, 48(3): pp209-224, 1994.

15.Molenaar M., "A Formal Data Structure For Three Dimensional Vector Maps", Proc $4^{\text {th }}$ International Symposium on spatial data handling, Zurich, 1990, pp830843.

16.Oosterom P.V., Reactive Data Structures for Geographic Information Systems, Oxford University Press, Oxford, 1993.

17.Peter R. G., Andrew J. B. "Three dimensional representation in a Geosicientfic Resource Management System for the minerals industry." Three-dimensional applications in Geographical Information Systems, Taylor \& Francis, Edit by Jonathan F. Raper, 1989, pp155-182.

18.Pfund M. "Topologic Data Structure for a 3D GIS". http://www.gis.ethz.ch/publications

19.Pigot S. "A topological model for a 3-dimensional spatial information system". PhD. thesis, University of Tasmania, 1998.

20.Pilouk M., Tempfli K., Molenaar M., "A Tetrahedron-Based 3D Vector Data Model for Geoinformation." In: Advanced Geographic Data Modelling, Netherlands Geodetic Commission, Publications on Geodesy, (40): ppl29-140, 1994.

21.Raper J. "The 3-dimensional geoscientific mapping and modelling system: a conceptual design." Three-dimensional applications in Geographical Information Systems, Taylor \& Francis, Edit by Jonathan F. Raper, 1989, pp11-19.

22.Stoter J. "Needs possibilities and constraints to develop a 3D cadastral registration system". http://www.geo.tudelft.nl/GISt/gist e/index.htm

23.Sun M., Chen Jun, Zhang X. Z. "3DCM data modelling based on surface partition". Acta Geodateica et Cartographica Sinica, Vol.29, No.3, 257-265, 2000.

24.Tempfle K. "3D topographic mapping for urban GIS". ITC journal 1998-3/4, 181190, 1998.

25.Turer A. K. "The role of three-dimensional geographic information systems in subsurface characterization for hydrogeological applications". Three-dimensional applications in Geographical Information Systems, Taylor \& Francis, Edit by Jonathan F. Raper, 1989, pp115-127.

26.Zlatanova S., Tempfli K. "Modelling for 3D GIS: Spatial Analysis and Visualisation through web". Proc of IAPRS, Vol. XXXIII, Amsterdam, 2000, pp1257-1264. 